\title{
Flujometría en la práctica de atención primaria
}

\author{
JUAN CÉSPEDES G., MÓNICA GUTIÉRREZ C., MANUEL OYARZÚN G. y COMISIÓN DE FUNCIÓN \\ PULMONAR DE ADULTOS, SOCIEDAD CHILENA DE ENFERMEDADES RESPIRATORIAS*
}

\section{Peak expiratory flow measurement in primary clinical practice}

Measurement of peak expiratory flow (PEF) by using the mini Wright meter is widely used for assessing patients with airway obstruction in the clinical practice. This document -written by the Pulmonary Function Commission of Chilean Respiratory Disease Society-provides a basic guideline for using this measurement in adult patients. Indications, technique and evaluation of the airway obstruction by measuring PEF through a mini Wrigth meter are briefly described.

Key words: Peak expiratory flow; mini Wright meter; airways obstruction; guideline.

\section{Resumen}

La medición del flujo espiratorio máximo (FEM) con el mini-flujómetro de Wright se usa ampliamente en la práctica clínica para evaluar pacientes con obstrucción de las vías aéreas. Este documento redactado por la Comisión de Función Pulmonar de la Sociedad Chilena de Enfermedades Respiratorias, proporciona una guía práctica para efectuar esta medición en pacientes adultos. En este artículo se describen resumidamente las indicaciones, la técnica y la evaluación de la obstrucción de las vías aéreas a través de la medición del FEM con el mini-flujómetro de Wright.

Palabras clave: Flujo espiratorio máximo; mini-flujómetro de Wright; obstrucción de vías aéreas: guía práctica.

\section{Introducción}

La utilización de la medición del Flujo Espiratorio Máximo (FEM) o flujo espiratorio tope o cúspide con un equipo diferente al tradicional espirómetro, ha sido posible con la creación del flujómetro de Wright y ulteriormente con su versión simplificada, conocida como "mini Wright".

Estos equipos, de menor costo y actualmente de amplia distribución, se utilizan en los enfermos con patologías respiratorias obstructivas, porque las variaciones de sus mediciones son paralelas a las del volumen espiratorio forzado en el primer segundo $\left(\mathrm{VEF}_{1}\right)$ en la espirometría, que ha sido la evaluación tradicional del diagnóstico y seguimiento de las limitaciones ventilatorias obstructivas.

\section{Indicaciones}

- Evaluación de patologías con alteraciones respiratorias, principalmente obstructivas.
- Evaluación de la variabilidad circadiana de sus resultados.

- Cuantificación de la gravedad de la alteración.

- Objetivación de la respuesta a terapias broncodilatadoras o esteroidales.

- Evaluación en el tiempo de la patología de base.

- Evaluación de la obstrucción bronquial por ejercicio.

\section{Técnica}

1. La persona debe estar en posición de pie.

2. Debe realizar una inspiración máxima.

3. Colocarse la boquilla en la boca.

4. Fijarla bien con los labios.

5. Espirar lo más fuerte y rápido posible, antes de 4 segundos después de haber hecho una inspiración máxima.

- La maniobra debe repetirse al menos tres veces, permitiendo un tiempo de descanso adecuado entre ellas.

\footnotetext{
* Comisión de Función Pulmonar de Adultos integrada por los Drs. MT. Beroíza W., G. Borzone T., C. Cartagena S., I. Caviedes S., J. Céspedes G., M. Gutiérrez C, M. Gutiérrez N., R. Moreno B., M. Oyarzún G, y P. Schönffeldt G.
} 
- Las dos mayores mediciones deben tener una diferencia menor a $20 \mathrm{~L} / \mathrm{min}$ entre ellas. Si no la hay, el paciente deberá seguir haciendo maniobras de espiración forzada, hasta un máximo de 8 .

- Se debe registrar el más alto valor obtenido en las mediciones y la hora del día en que se efectuó la medición.

Hay que tener presente que se trata de maniobras cuyo resultado (FEM) es dependientes del esfuerzo y que en pacientes nuevos es necesario explicar bien la técnica y entrenarlos antes de efectuar el registro.

Los equipos tienen una vida media útil que es muy dependiente del cuidado que se tenga con ellos, por lo cual se aconseja comparar sus resultados cada 3 meses con controles biológicos (3 personas con FEM conocido, sanas, no fumadoras, de diferentes tallas).

La periodicidad de las mediciones en pacientes obstructivos depende de la gravedad o inestabilidad de su patología. Puede ser diaria y en 3 momentos diferentes (mañana, tarde y noche) o semanal, quincenal o mensual.

Lo mínimo aceptable es que el FEM se mida SIEMPRE cuando el paciente asiste a control e idealmente a la misma hora del día.

\section{Evaluación}

Se debe usar siempre el gráfico de valores normales de Gregg y Nunn ${ }^{1}$, no es conveniente extrapolar valores de la espirometría.
Idealmente se debiera tener conocido y registrado el "mejor valor" en los pacientes crónicos, para usarlos como referencia.

1. Si se obtiene un valor de FEM igual o superior al $90 \%$ del teórico o mejor valor conocido, se considera como patología estable o controlada.

2. Si el valor del FEM es inferior a dicho valor, hay que considerar al paciente con patología fuera de control.

3. Si el valor del FEM es inferior a $150 \mathrm{~L} / \mathrm{min}$ es signo de gravedad y probable requerimiento de hospitalización, salvo que el mejor valor conocido sea cercano.

$\underset{\text { diaria }}{\operatorname{Variabilidad}}=\frac{\text { FEM mayor }- \text { FEM menor }}{\text { FEM mayor }} \cdot 100$

No debe ser mayor a $20 \%$, pues reflejaría inestabilidad.

Obstrucción por ejercicio $=$

FEM mayor - FEM menor 6 min post ejercicio $\bullet 100$ FEM mayor

Es positivo si es mayor a $15 \%$.

\section{Bibliografías}

1.- GREGG I, NUNN A J. New regression equations for predicting peak expiratory flow in adults Brit Med J 1989; 298: 1068-70.

2.- SEPÚLVEDA R. El flujómetro de Wright. Una herramienta indispensable en la práctica ambulatoria. Rev Chil Enf Respir 2004; 20: 80-4. 